\title{
32-core Dense SDM Unidirectional Transmission of PDM- 16QAM Signals Over 1600 km Using Crosstalk-managed Single-mode Heterogeneous Multicore Transmission Line
}

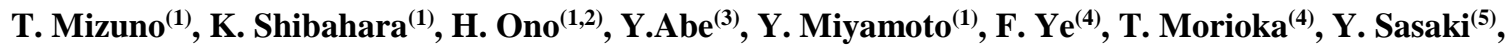 \\ Y. Amma ${ }^{(5)}$, K. Takenaga ${ }^{(5)}$, S. Matsuo ${ }^{(5)}$, K. Aikawa ${ }^{(5)}$, K. Saitoh ${ }^{(6)}$, Y. Jung ${ }^{(7)}$, D. J. Richardson ${ }^{(7)}$, \\ K. Pulverer ${ }^{(8)}$, M. Bohn ${ }^{(8)}$, and M. Yamada ${ }^{(9)}$ \\ ${ }^{(1)}$ NTT Network Innovation Laboratories, NTT Corporation, 1-1 Hikari-no-oka, Yokosuka, Kanagawa, 239-0847 Japan \\ ${ }^{(2)}$ NTT Device Technology Laboratories, NTT Corporation, 3-1 Morinosato-Wakamiya, Atsugi, Kanagawa, 243-0198 Japan \\ ${ }^{(3)}$ NTT Device Innovation Center, NTT Corporation, 3-1 Morinosato-Wakamiya, Atsugi, Kanagawa, 243-0198 Japan \\ (4) Technical University of Denmark, DK2800 Kgs. Lyngby, Denmark \\ (5) Optics and Electronics Laboratory, Fujikura Ltd., 1440, Mutsuzaki, Sakura, Chiba, 285-8550 Japan \\ ${ }^{(6)}$ Hokkaido University, North 14 West 9, Sapporo, Hokkaido, 060-0814 Japan \\ ${ }^{(7)}$ Optoelectronics Research Centre, University of Southampton, Southampton, SO17 1BJ, UK \\ ${ }^{(8)}$ Coriant GmbH \& Co. KG, Munich, Germany \\ (9) Osaka Prefecture University, 1-1 Gakuen-cho, Sakai, Osaka 599-8531, Japan \\ Authore-mail address: mizuno.takayuki@lab.ntt.co.jp
}

\begin{abstract}
We demonstrate a 32-core dense space division multiplexed (DSDM) transmission of PDM-16QAM 20-WDM signals over $1644.8 \mathrm{~km}$ with a record aggregate SE distance product of $331,300 \mathrm{~b} / \mathrm{s} / \mathrm{Hz} \cdot \mathrm{km}$ employing a low-crosstalk 32-core fiber in a partial recirculating-loop system.

OCIS codes: (060.2330) Fiber optics communications; (060.1660) Coherent communications
\end{abstract}

\section{Introduction}

Space division multiplexing (SDM) using multicore and multimode fiber [1] has shown excellent potential as a means to develop ultra-high capacity optical fiber transmission systems [2-5]. Utilizing both multiple cores and multiple modes has allowed dense SDM (DSDM) transmission leading to increases in aggregate spectral efficiency (SE) per fiber to over several hundred $\mathrm{b} / \mathrm{s} / \mathrm{Hz}$ [6], and spatial multiplicities in excess of 100 [7,8]. However, the transmission distances in such studies have been less than around $500 \mathrm{~km}$ for 36 multiplicity [9] and less than around $10 \mathrm{~km}$ for over 100 multiplicity [7,8]. Figure 1 shows the spatial multiplicity and aggregate SE versus distance of recent SDM-WDM experiments. Several SDM studies have presented long-haul transmission of over $1000 \mathrm{~km}$, including a 1000-km and 1050-km 3-mode transmission [10,11], 1500-km 12-core bi-directional (6-core unidirectional) transmission [12], 1705-km 6 coupled-core transmission [13], and 7326-km 7-core transmission [14]. These long-haul experiments had only been realized with a limited spatial multiplicity, namely, a maximum of seven using a 7-core fiber [14]. To achieve a thousand-km transmission with a greater number of cores in a multicore fiber it is necessary to overcome the tradeoff between low crosstalk, high core-count, and large effective area in the limit of an acceptable cladding diameter in reliability terms.

In this paper, we report a record 32-core long distance DSDM transmission of over $1644.8 \mathrm{~km}$ achieved using a crosstalk-managed transmission line consisting of a novel partial recirculating loop system and a 51.4-km singlemode heterogeneous 32-core fiber with a $243 \mu \mathrm{m}$ cladding diameter, and low inter-core crosstalk of $<-39.4$ $\mathrm{dB} /$ span. We have successfully transmitted 32 spatial channels each containing 20 PDM-16QAM WDM channels at 12-Gbaud and $12.5 \mathrm{GHz}$-spacing over $1644.8 \mathrm{~km}$ with an SE of $6.295 \mathrm{~b} / \mathrm{s} / \mathrm{Hz}$, yielding to the best of our knowledge the highest ever reported aggregate SE distance product of $331,300 \mathrm{~b} / \mathrm{s} / \mathrm{Hz} \cdot \mathrm{km}$. This is 1.6 times larger compared to the previous record set in [14].
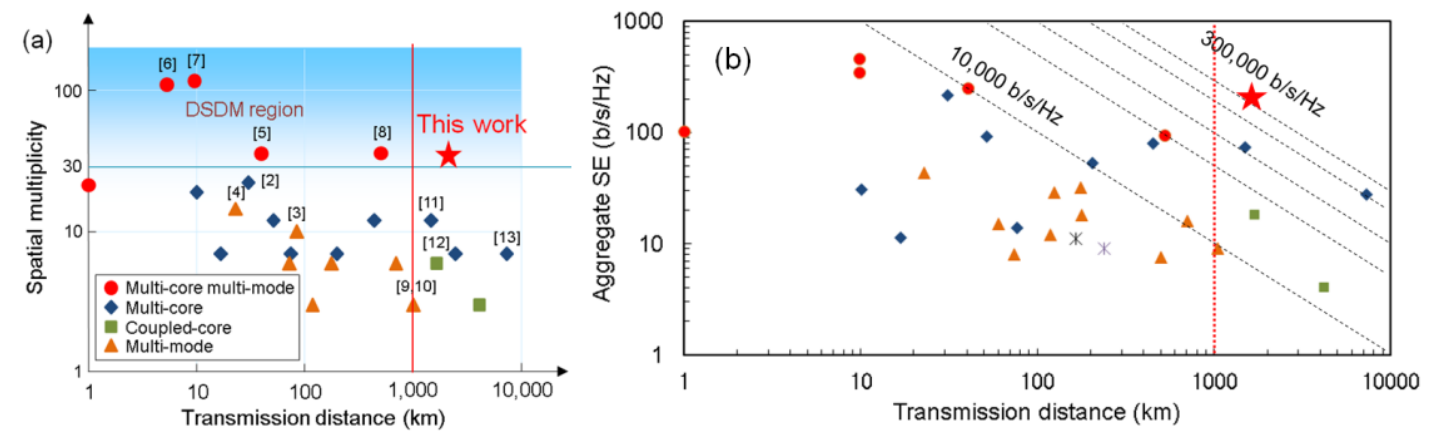

Fig. 1. (a) Spatial multiplicity, and (b) aggregate SE versus distance from recent SDM-WDM experiments. 


\section{Crosstalk-managed multicore transmission line}

To realize a DSDM transmission line for long-haul transmission, we designed a heterogeneous multicore fiber with two types of trench-assisted cores having high (type1, odd numbered cores) and low (type 2, even numbered cores) refractive index difference, and placed them in a square lattice arrangement as shown in Fig. 2(a). The core pitch is $29 \mu \mathrm{m}$, and the cladding diameter is $243 \mu \mathrm{m}$. We fabricated a single spool of 51.4-km long 32-core fiber. The effective area of each core was $>80.3 \mu \mathrm{m}^{2}$, and the measured loss at $1550 \mathrm{~nm}$ was $<0.24 \mathrm{~dB} / \mathrm{km}$. The worst crosstalk is $<-39.4 \mathrm{~dB} /$ span when light is launched into four adjacent cores of the same refractive index type in the diagonal positions (Fig.2(b)), and $<-54 \mathrm{~dB} /$ span using four adjacent cores of the different refractive index type (Fig.2(c)). This result shows that inter-core crosstalk arises mostly from cores with the same refractive index type (Fig.2(b)), and is effectively reduced by the heterogeneous design. Thus, even with a dense core arrangement and the cladding diameter of typical previous MCFs, our heterogeneous 32-core fiber offers sufficient effective area and inter-core crosstalk suitable for long-haul transmission.

(a)

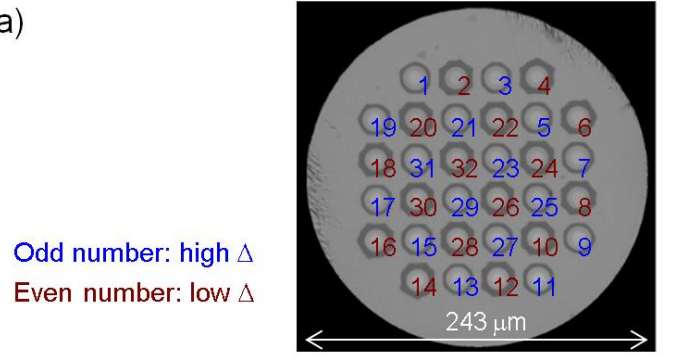

(b)

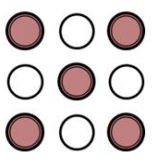

Four adjacent cores of the same refractive index type (c)

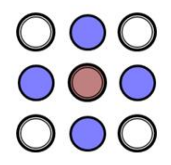

Four adjacent cores of the different refractive index type

Fig. 2. (a) 32-core MCF cross-section, and adjacent cores of the (b) same and (c) different refractive index type.

We developed the partial recirculating loop system shown in Fig. 3(a) to evaluate the long-haul transmission characteristics of a high core-count fiber. The system is loaded with four parallel recirculating signals to adjacent cores of the same refractive index type as indicated in Fig.2(b), which will affect the core under test by inter-core crosstalk. The remaining 27 cores are loaded with non-recirculating signals, which will have negligible effect on the signal of the core under test because of the low crosstalk. We configured the experimental setup as such to increase the degree of approximation of a full recirculating loop system. This measurement technique is believed to be effective, in particular, for measuring transmission characteristics of a fiber having more than several tens of cores.

\section{Long-haul DSDM transmission experiment}

20-ch 12.5-GHz-spaced 96-Gb/s PDM-16QAM signals were generated at the transmitter and split into three branches. The first branch was used for the core under measurement (loop \#1), the second branch was further split into four and these were used to provide recirculating crosstalk signals (loops \#2-\#5). The third branch was further split into 27, and these signals were used as non-recirculating signals. All 16QAM WDM signals were digitally generated, each driven at 12 Gbaud and reshaped by a root-raised-cosine filter with 0.01 roll-off factor and independent pieces of pseudo-random-binary-sequence (PRBS) of length $2^{23}-1$. A frame length of 31250 symbols

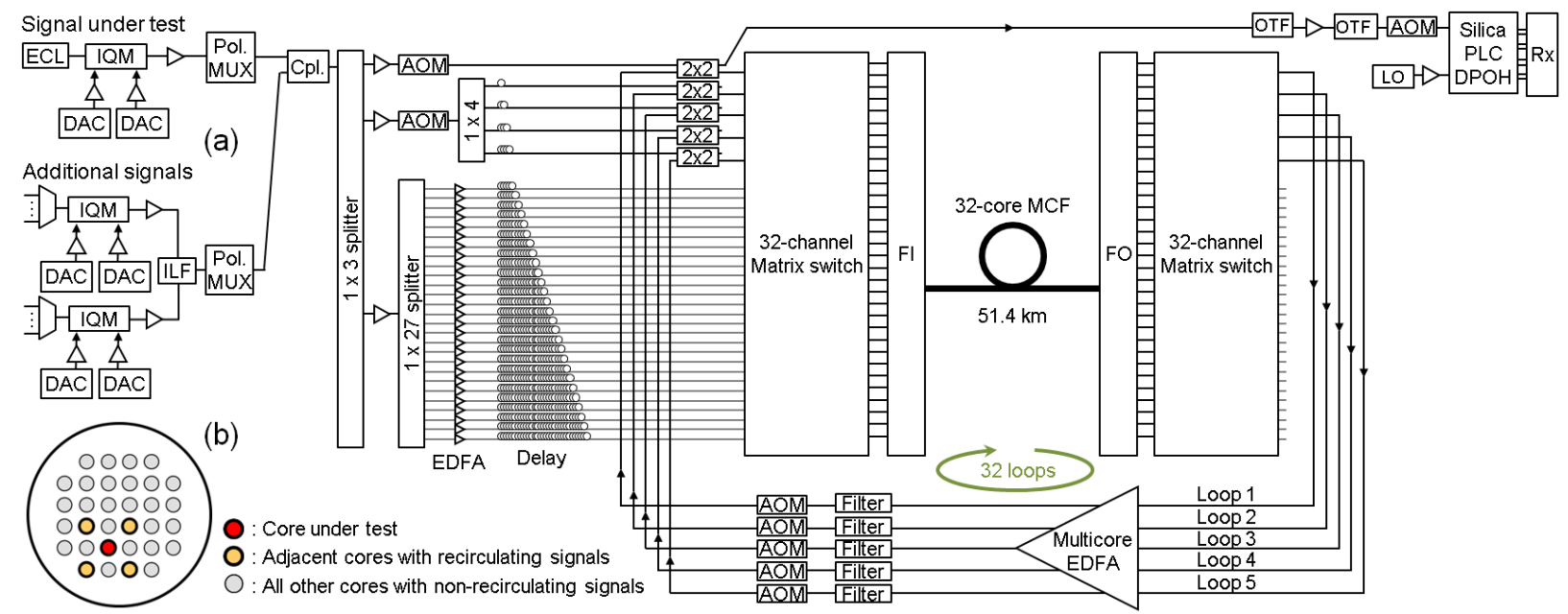

Fig. 3. (a) Experimental setup, and (b) example signal allocation when core \#28 is under test. 
comprised a payload, $1.63 \%$ overhead for training sequence, and $20 \%$ overhead for forward error correction (FEC). The net data rate was $78.69 \mathrm{~Gb} / \mathrm{s}$, the SE was $6.295 \mathrm{~b} / \mathrm{s} / \mathrm{Hz} /$ core, and the aggregate SE was $201.46 \mathrm{~b} / \mathrm{s} / \mathrm{Hz}$. The signal under test was generated using a tunable external-cavity laser (ECL) with $\sim 60-\mathrm{kHz}$ linewidth, the remaining channels were generated using DFB lasers with 2-MHz linewidths. Odd and even wavelength channels around 1549.1-1551.0 nm were multiplexed, separately modulated by IQMs, and combined by a $12.5 / 25 \mathrm{GHz}$ interleave filter. The signal under test and the remaining signals were each polarization division multiplexed (PDM) by a PDM emulator with a $10 \mathrm{~ns}$ delay, and combined with a coupler. The 32 signals were relatively delayed by $0, \Delta \mathrm{t}, 2 \Delta \mathrm{t}, \ldots$, and $31 \Delta \mathrm{t}$ with a unit time delay $\Delta \mathrm{t}$ of $20 \mathrm{~ns}$ for decorrelation of signals between the 32 cores. The optical power for the 32 spatial channels were all set at $-10 \mathrm{dBm} /$ wavelength/core at the input of the multicore fiber.

The partial recirculating loop system consisted of 32-channel matrix switches, a 51.4-km spool of the 32-core fiber, 32-channel free space optics based fan-in/fan-out (FI/FO) devices, and five parallel recirculating loops. Signals in the loops were amplified by five channels of a seven-core erbium-doped fiber amplifier (EDFA). The 32channel matrix switches were used to select cores and cross-connect the paths of the input and output ports of the transmission line for each measurement as described above. For example, when core \#28 was under measurement, cores \#28, \#12, \#14, \#26, and \#30 were selected to form recirculating loops \#1-\#5, respectively, and recirculated for 32 loops, while the remaining 27 cores were loaded with non-recirculating signals. The same operations were performed for all 32 cores.

The signal from the core under test was filtered by tunable optical filters and input to a planar lightwave circuit (PLC) type dual polarization optical hybrid (DPOH). It was then digitized at $40 \mathrm{GS} / \mathrm{s}$ using a 4-ch digital storage oscilloscope and stored in sets of 4M samples. In the receiver-side offline processing, frond-end error correction, chromatic dispersion compensation, and frequency-offset cancellation were first performed. Then, equalization was carried out using $64 T / 2$-spaced-taps LMS-based $2 \times 2$ frequency domain equalization (FDE) combined with phase recovery. $0.75 \mathrm{M}$ bits were used to calculate BERs and Q-factors.

Figure 4 (a) shows the measured Q-factors for all channels as a function of the core number after $1644.8 \mathrm{~km}$ transmission (32 loops). The measured Q-factors for all 32 cores for the 20 wavelengths exceeded the Q-limit of 5.7 dB with $20 \%$ FEC overhead. Example constellations of core \#16, wavelength \#11 are shown in Fig. 4(b).

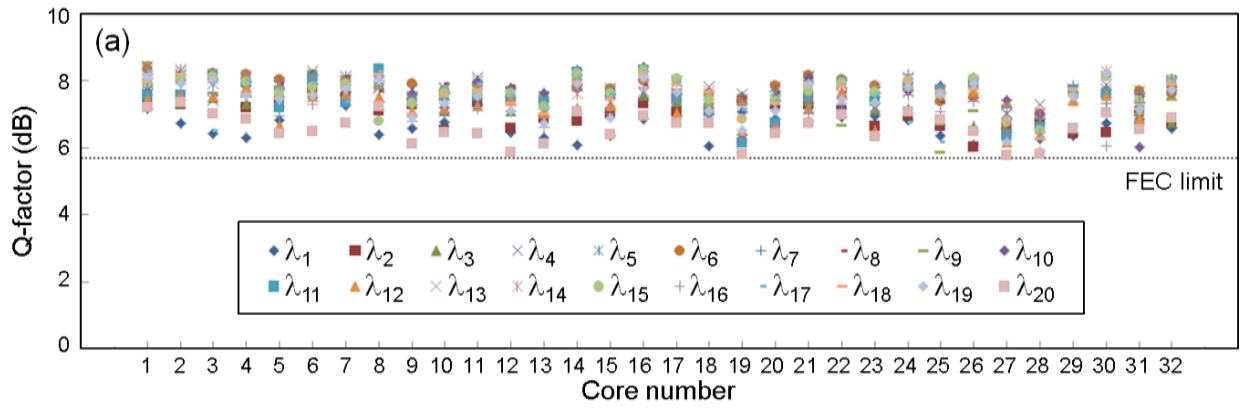

(b)

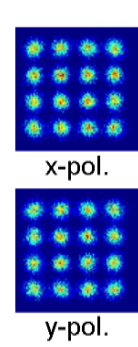

Fig. 4. Measured (a) Q-factors and (b) example constellations after $1644.8 \mathrm{~km}$ transmission.

\section{Conclusions}

We have demonstrated a record 32-core DSDM transmission over $1644.8 \mathrm{~km}$ of 20-WDM PDM-16QAM signals with a record aggregate SE distance product of $331,300 \mathrm{~b} / \mathrm{s} / \mathrm{Hz} \cdot \mathrm{km}$. The distance is more than three times longer for DSDM with a spatial multiplicity of 32, and the aggregate SE distance product is more than 1.6 times larger than previously reported record. The results were achieved using a crosstalk-managed transmission line consisting of a novel partial recirculating loop system and a 51.4-km single-mode heterogeneous 32-core fiber with a $243 \mu \mathrm{m}$ cladding diameter, and low inter-core crosstalk of $<-39.4 \mathrm{~dB} / \mathrm{span}$.

Part of this research utilized results from the EU-Japan coordinated R\&D project on "Scalable And Flexible optical Architecture for Reconfigurable Infrastructure (SAFARI)" commissioned by the Ministry of Internal Affairs and Communications (MIC) of Japan and EC Horizon 2020, and research commissioned by the National Institute of Information and Communications Technology (NICT) of Japan.

\section{References}

[1] T. Morioka, OECC2009, FT4 (2009).

[2] B. J. Puttnam et al., ECOC2015, PDP3.1 (2015).

[3] R. Ryf et al., ECOC2015, PDP3.3 (2015).

[4] M. Arikawa et al., ECOC2015, Th.1.2.3 (2015).

[5] N. K. Fontaine et al., OFC2015, Th5C.1 (2015).

[6] T. Mizuno et al., OFC2014, Th5B.2 (2014).

[7] J. Sakaguchi et al., OFC2015, Th5C.2 (2015).

[8] D. Soma et al., ECOC2015, PDP.3.2 (2015).

[9] K. Shibahara et al., OFC2015, Th5C.3 (2015).

[10] E. Ip et al., OFC/NFOEC2013, PDP5A.2 (2013).

[11] R. Ryf et al., ECOC2015, Tu.3.2.3 (2015).

[12] T. Kobayashi et al., ECOC2013, PD3.E.4 (2013).

[13] R. Ryf et al., ECOC2014, PD.3.2 (2014).

[14] K. Igarashi et al., ECOC2013, PD3.E.3 (2013). 